Artículo Original

\title{
Impacto funcional y psicosocial del uso de prótesis de bajo costo en impresión 3D en amputado unilateral de antebrazo: estudio de un caso
}

LORENA LLORENTE D ${ }^{1}$, DENISSE SOTO R ${ }^{1}$, SERGIO REYES B ${ }^{1}$, JORGE ZÚÑIGA U².

'Instituto Teletón Concepción, Chile. ${ }^{2}$ Department of Biomechanics, University of Nebraska at Omaha, EUA.

Los autores declaran no tener conflictos de interés.

Recibido: 30-11-2018 Aceptado: 29-04-2019

Correspondencia a: Sergio Reyes Burgos sreyes@teleton.cl

\section{ABSTRACT \\ Functional and psychosocial impact of the use of low cost 3D prosthesis in a subject with unilateral forearm amputation: Case report}

Introduction: The range of prosthetic devices (hooks and cosmetic hands) is limited for transradial amputation patients. Having new low-cost 3D prosthetic systems available, as well as experience on how to use them, will increase the options for better functionality, activity and participation. Objective: To assess functional and psychosocial impact of the use of 3D printed prosthetic hand. Method: A 15 year-old Teleton patient was selected with a middle third transradial amputation of the left forearm; informed consent was obtained. A plaster mold was taken and a 3D prosthesis was made using acrylonitrile butadiene styrene (ABS) and polylactic acid (PLA); the thumb had two articulations and the other fingers had three articulations. The prosthesis comprised also forearm and wrist. The user attended four prosthetic training sessions in a month. Modified Bilan 400 points scale for hand functionality, upper extremity functional index (UEFI) and the psychosocial impact of assistive devices scale (PIADS) were applied before admittance, and then at three and eleven months of follow up. Results: Hand functionality showed an increase of approximately $30 \%$ with both measuring instruments. In case of PIADS, competence, self-esteem and adaptability varied, from no effect of technology assistance, to the maximum of 3 points in adaptability at the eleven-month follow up. Conclusion: This case study highlights potential benefits of using upper limb 3D prosthesis on daily functional performance and quality of life.

Key words: Amputees, prosthesis, 3D printing, upper extremity functionality, quality of life.

\section{RESUMEN}

Introducción: Los pacientes que presentan una amputación a nivel transradial cuentan con oferta reducida de dispositivos protésicos (gancho y mano cosmética). Postulamos que disponer de nuevos sistemas protésicos $3 \mathrm{D}$ de bajo 
costo y la experiencia de su uso, permitirá aumentar las opciones para mejorar funcionalidad, actividad y participación. Objetivo: Evaluar el impacto funcional y psicosocial del uso de mano protésica manufacturada con impresión 3D. Método: Se seleccionó un paciente Teletón con amputación transradial de antebrazo izquierdo nivel tercio medio, 15 años de edad, con consentimiento informado. Se tomó molde en yeso y confeccionó prótesis 3D fabricada en acrilonitrilo butadieno estireno (ABS) y ácido poli-láctico (PLA); el pulgar tiene dos articulaciones y los demás dedos tres articulaciones. La prótesis la constituyen también el antebrazo y muñeca. El usuario recibió cuatro sesiones de entrenamiento con la prótesis en el lapso de un mes. Las evaluaciones con pauta de funcionalidad de mano Bilan 400 points modificada, índice funcional de las extremidades superiores (UEFI) y escala del impacto psicosocial de la asistencia tecnológica (PIADS), se aplicaron previo al ingreso, a tres y once meses de seguimiento. Resultados: La funcionalidad de mano mostró un incremento de alrededor de 30\% con ambos instrumentos. En medición PIADS, competencia, autoestima y adaptabilidad, variaron de ningún efecto de la asistencia tecnológica, hasta el máximo de 3 puntos para adaptabilidad en el seguimiento a 11 meses. Conclusión: Este estudio de caso destaca potenciales beneficios del uso de prótesis de extremidad superior en el desempeño funcional y calidad de vida.

Palabras clave: Amputados, prótesis, impresión tridimensional, funcionalidad, extremidades superiores, calidad de vida.

\section{Introducción}

Las estimaciones mundiales para las amputaciones totales y/o parciales de las extremidades superiores varían de $4-5$ por 10.000 a 1 por 100 nacidos vivos ${ }^{1}$. En Institutos Teletón Chile, de los 27.912 niños y adolescentes atendidos, $5,7 \%$ tienen el diagnóstico de amputaciones y/o malformaciones de extremidades de origen congénito.

Los requerimientos protésicos de estos pacientes son variados, considerando aspectos como: el largo relativo del muñón, el potencial de crecimiento, las funciones por suplir (especialmente la sensitiva), el sistema de generación de potencia, el diseño cosmético y la repercusión que tiene en su desarrollo psicosocial $^{2}$.

Las prótesis 3D también parecen tener un impacto psicológico y social para los niños de 6 a 8 años de edad, así como pueden tener un potencial significativo para afectar positivamente la calidad de vida y el uso diario ${ }^{3,4}$. No obstante lo anterior, no se encontraron estudios que indiquen o respalden el impacto psicosocial del uso de prótesis $3 \mathrm{D}$.

Unidades electrónicas (mioeléctrica) y dispositivos mecánicos (ganchos metálicos) han sido mejorados constantemente para dar respuestas a requerimientos de los pacientes (por ejemplo: comodidad, durabilidad y control); no obstante, el costo de mantenimiento y reposición sigue siendo elevado, dificultando el acceso a ellas ${ }^{5}$. Aunque responden a ciertos requerimientos, su confección es compleja y su diseño no mejora aspectos cosméticos, los cuales no otorgan aún una apariencia más atractiva, lo que aumenta la tasa de rechazo e insatisfacción a su uso ${ }^{6,7}$.

Estudios internacionales actuales evidencian que el uso de dispositivos protésicos eléctricos y/o mecánicos, en pacientes amputados, junto a programas de apoyo multidisciplinarios, aumentan la destreza en el manejo de la prótesis, mejorando su esquema corporal, lateralidad, coordinación bimanual y óculo-manual, motricidad gruesa y fina, al tiempo que desarrollan un adecuado ritmo y expresión corporal ${ }^{6}$. 
Asimismo, las prótesis pueden facilitar la aceptación social o pueden ser útiles como herramientas para actividades especializadas ${ }^{8}$.

En relación a lo anterior, el objetivo del estudio es evaluar el impacto funcional y psicosocial del uso de la mano protésica manufacturada con impresión 3D.

\section{Metodología}

\section{a) Información del paciente}

Paciente que fue seleccionado aleatoriamente, desde cubo OLAP (On-line Analytical Processing), de Instituto Teletón Concepción, sexo masculino, quince años, prematuro de 33 semanas; sufrió amputación por causa vascular en periodo neonatal en tercio medio de antebrazo izquierdo, actualmente muñón transradial de $12,5 \mathrm{~cm}$, cónico, con fuerza muscular de flexores y extensores de brazo normal, de acuerdo a escala Medical Research Council ${ }^{9}$. Paciente inserto en familia nuclear compuesta por ambos padres y dos hermanos menores. De acuerdo a evaluación de rutina realizada por asistente social del equipo de clínica del paciente, no se visualizaron factores de riesgo psicosociales. Cursaba $1^{\circ}$ año de enseñanza media, bien integrado, independiente en actividades de la vida diaria. En etapa de lactante, usó prótesis tipo mitón; posteriormente, prótesis con gancho de uso intermitente. Al momento del estudio, no la usaba.

\section{Elaboración de prótesis}

Se confeccionó prótesis 3D, fabricada en acrilonitrilo butadieno estireno (ABS) y ácido poli-láctico (PLA), con un peso total de 690 gramos, 50 gramos más que la prótesis tradicional. La prótesis la constituyeron, además, el antebrazo y la muñeca, sin movimiento articulatorio de esta última, todos los dedos tenían articulación (el pulgar dos articulaciones y los demás dedos tres articulaciones). El agarre estaba basado en sistema de pinza, mediante el dedo pulgar en oposición al dedo índice. Todos los dedos tenían un recubrimiento de silicona para mejorar la adherencia.

El mecanismo de accionamiento para el movimiento de los dedos era a través de cin- co cables en los dedos unidos a un cable que se conectaba con un tensor de regulación en sistema BOA. Este sistema activaba el cierre voluntario de la mano protésica con un mecanismo de polea y la extensión pasiva se lograba con componentes elásticos; con la flexión de codo se generaba el cierre de la mano y con la extensión de codo, la apertura de la mano (Figuras 1 y 2).

\section{b) Método de evaluación funcional y social}

Se utilizó pauta de funcionalidad de mano Bilan 400 points modificada ${ }^{10}$ y el índice funcional de extremidades superiores (UEFI) ${ }^{11}$, ambas medidas validadas y confiables como instrumentos evaluativos de funcionalidad, lo

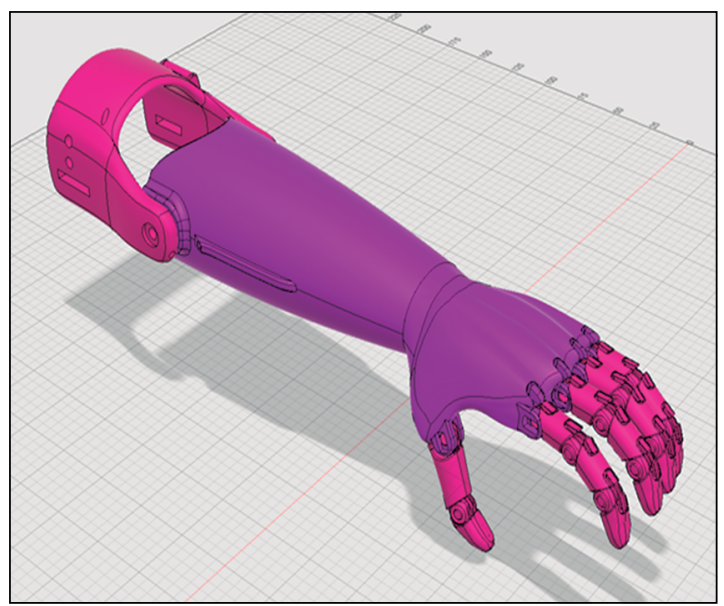

Figura 1. Mano en extensión-software de diseño 3D.

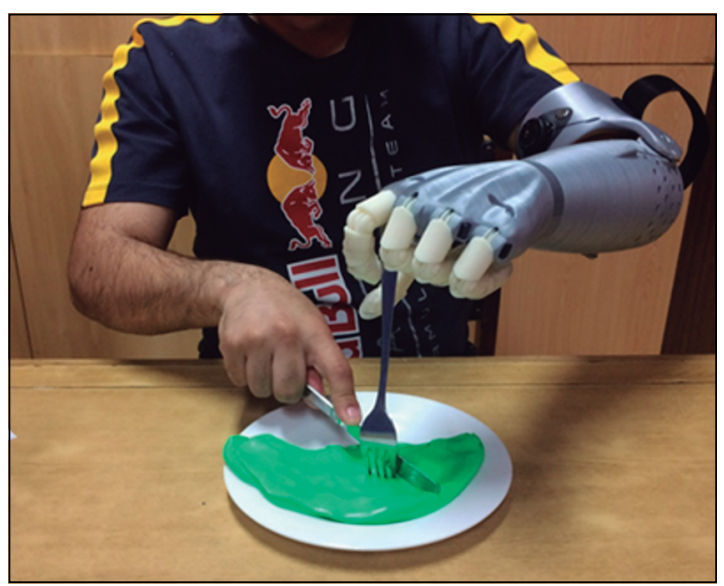

Figura 2. Mano en flexión usada por paciente en actividad bimanual. 
que permitió objetivar las intervenciones en rehabilitación.

Para Bilan, en la dimensión III, la escala comprende diecinueve actividades funcionales con una valoración posible de cero puntos (la actividad no se realiza) a tres puntos (la actividad es realizada con movimiento normal), con un máximo posible de 57 puntos.

UEFI, comprende veinte actividades funcionales con una valoración posible de cero puntos (la actividad no se realiza) a cuatro puntos (la actividad es realizada sin dificultad), con un máximo posible de 80 puntos.

Para la escala Bilan, sus autores no hacen referencia al puntaje mínimo requerido para considerar que el cambio sea de significancia clínica.

En cuanto a UEFI, sus autores señalan que la diferencia positiva mínima clínicamente importante es de 8 puntos sobre 80 .

En evaluación del impacto psicosocial, se utilizó la escala del impacto psicosocial de la asistencia tecnológica (PIADS) ${ }^{12}$, la que ha demostrado fiabilidad y validez para estas mediciones. Esta escala permite evaluar los efectos de un dispositivo de asistencia en ámbitos de competencia, adaptabilidad y autoestima y la repercusión de estas en la calidad de vida. PIADS consta de tres dimensiones, con un total de veintiséis ítems a evaluar, con una valoración posible de -3 puntos (ha disminuido la dimensión estudiada) a tres puntos (la dimensión estudiada ha aumentado). Entre ambas valoraciones se encuentra la opción cero, que se aplica cuando el usuario no está seguro sobre qué puntuación aplicar o decide que la asistencia tecnológica no tiene efecto alguno.

En cuanto a la significancia clínica de los resultados para la PIADs, sus autores se refieren a la obtención de puntajes positivos, que implican un impacto positivo en la calidad vida. Si los puntajes son negativos, significa lo contrario.

Todas las pautas se aplicaron en estado basal (sin la prótesis), a los tres meses de entregada la prótesis $\mathrm{y}$, finalmente, a los once meses de seguimiento.

El tiempo de uso diario de la prótesis 3D, en promedio fue de 5 horas. Las actividades seleccionadas para realizar con la prótesis fueron escogidas por el usuario de acuerdo a su criterio. Tanto las actividades realizadas como el promedio de uso diario de la prótesis, fueron las mismas a los tres y 11 meses de uso. La transportó al colegio, utilizándola para realizar actividades que requirieron bimanualidad en asignaturas como arte, tecnología y talleres deportivos (tenis de mesa), usándola en promedio 3,5 horas durante su jornada escolar. En casa utilizó la prótesis para actividades como preparar la comida y comer con utensilios. Además, fue capaz de andar en bicicleta y hacerle la mantención con ella. El tiempo de uso en casa fue en promedio 1,5 horas.

\section{Resultados}

Las actividades funcionales evaluadas mediante dimensión III de pauta Bilan, mostraron un incremento del puntaje de $30,6 \%$ entre la evaluación basal y los tres meses, aumentando algo más al finalizar la experiencia (Tabla 1).

La evaluación de las actividades funcionales mediante pauta UEFI mostró una ganancia de $30 \%$ entre evaluación basal y los tres meses, ganancia que se mantuvo al finalizar el seguimiento (Tabla 2).

La evaluación del impacto psicosocial mediante pauta PIADS, mostró un incremento de 2,5 puntos en promedio en los ámbitos

Tabla 1. Funcionalidad mediante pauta Bilan 400 points modificada

\begin{tabular}{|c|c|c|c|c|c|}
\hline \multirow[t]{3}{*}{ Pauta Bilan 400 points* } & \multirow[t]{2}{*}{ Basal } & \multirow[t]{2}{*}{3 meses } & \multirow[t]{2}{*}{11 meses } & \multicolumn{2}{|c|}{ Ganancia** } \\
\hline & & & & 3 meses & 11 meses \\
\hline & ptje & ptje & ptje & ptje $\%$ & ptje $\%$ \\
\hline Dimensión III funcionalidad & $36(63,1)$ & $47 \quad(82,4)$ & $48 \quad(84,2)$ & $11(30,6)$ & $12(33,3)$ \\
\hline
\end{tabular}

*Puntaje máximo: 57 puntos. **\% Ganancia= ptje final - ptje inicial / ptje inicial. 
Tabla 2. Funcionalidad mediante Índice funcional de extremidades superiores UEFI

\begin{tabular}{|c|c|c|c|c|c|}
\hline \multirow[t]{3}{*}{ Evaluación UEFI } & \multirow[t]{2}{*}{ Basal } & \multirow[t]{2}{*}{3 meses } & \multirow[t]{2}{*}{11 meses } & \multicolumn{2}{|c|}{ Ganancia } \\
\hline & & & & 3 meses & 11 meses \\
\hline & ptje & ptje & ptje & ptje \% & ptje $\%$ \\
\hline $\begin{array}{l}\text { Índice funcional de extremidades } \\
\text { superiores }\end{array}$ & $52 \quad(65,0)$ & $68 \quad(85,0)$ & $68(85,0)$ & $16(30,7)$ & $16(30,7)$ \\
\hline
\end{tabular}

*Puntaje máximo: 80 puntos. **\% Ganancia= ptje final - ptje inicial / ptje inicial.

Tabla 3. Impacto psicosocial de la asistencia tecnológica con evaluación PIADS

\begin{tabular}{|lccc|}
\hline $\begin{array}{l}\text { Evaluación } \\
\text { PIADs }\end{array}$ & $\begin{array}{c}\text { Basal } \\
\text { ptje }\end{array}$ & $\begin{array}{c}\mathbf{3} \text { meses } \\
\text { ptje }\end{array}$ & $\begin{array}{c}\mathbf{1 1} \text { meses } \\
\text { ptje }\end{array}$ \\
\hline Competencia & 0 & 2,17 & 2,83 \\
Adaptabilidad & 0 & 2,83 & 3,00 \\
\hline Autoestima & 0 & 2,00 & 2,63 \\
\hline
\end{tabular}

estudiados a los 3 meses de seguimiento, lo que corresponde a más de $80 \%$ del máximo posible de la escala. A los 11 meses, la adaptabilidad fue la dimensión que presentó una mayor ganancia respecto de la evaluación basal (Tabla 3), destacando que en las 3 áreas se observó un incremento adicional.

\section{Discusión}

Los resultados encontrados indican que el uso de mano protésica con impresión $3 \mathrm{D}$, no motorizada, para amputaciones transradiales, puede mejorar el desempeño del usuario en el uso de sus extremidades superiores, de acuerdo a los resultados positivos en pautas de evaluación funcional UEFI, Bilan, así como también la pauta de impacto psicosocial de la asistencia tecnológica PIADS.

Observamos que las mejorías funcionales que mostró el paciente con el uso de la prótesis, tienen relación con el adecuado control del dispositivo, confortabilidad, aspectos cosméticos y funcionalidad de esta. Destaca de igual forma, la motivación y adherencia mostrada por el usuario y su familia en todo el proceso terapéutico desarrollado.

La mejoría se visualiza especialmente al poder realizar nuevas actividades que requirieron participación de ambas manos, como revelan los resultados observados en Bilan y UEFI; esto explicaría el incremento en el nivel de su calidad de vida (resultados observados en PIADs), especialmente en adaptabilidad.

En cuanto a la significancia clínica de los resultados observados, UEFI arroja una ganancia de 16 puntos a los tres meses que se mantuvo a los once meses. Esto implica clínicamente un cambio significativo, siendo el cambio mínimo relevante 8 puntos.

Para Bilan, si se estima un 5\% de ganancia como clínicamente significativa, también se logró ésta, pues la ganancia fue superior al 30\%.

En cuanto a la PIADs, se observaron cambios solo en el sentido de puntajes positivos, lo que indica un impacto positivo en la calidad de vida en todos los ítems evaluados.

Cabe mencionar, como antecedente relevante, que durante todo el proceso, desde la primera prueba hasta la actualidad, no se detectaron eventos adversos en la salud del paciente derivado del uso de la prótesis misma. El muñón y el brazo no han mostrado señales de afección alguna, ni lesiones, heridas cutáneas o molestias musculares.

No obstante lo anterior, revisiones disponibles en la literatura mundial, muestran resultados no concluyentes en relación a los resultados funcionales y eventual impacto psicosocial del uso de prótesis 3D para amputados en miembros superiores, respecto de su condición clínica previa ${ }^{13}$. Consideramos que el éxito que se logre con el uso de prótesis para extremidad superior y mano con tecnología 3D depende de múltiples factores, como el diseño y tipo de activación usado, el paciente y sus expectativas. La revisión de Ten ${ }^{13}$ destaca la falta de estudios y evidencia especialmente 
en cuanto a la aceptación, funcionalidad y durabilidad de las manos 3D. Este reporte de caso ya abarca esos aspectos. Nuestra experiencia, además, nos indica que es relevante la selección del paciente, de modo que se ajusten las posibilidades que le ofrece este tipo de prótesis a las necesidades funcionales y estéticas del paciente.

El análisis de este caso plantea la necesidad de seguir incorporando pacientes a la experiencia del uso de una prótesis con impresión 3D.

La continuación de esta línea de investigación nos permitiría aumentar la casuística y mejorar aspectos del dispositivo, que en las evaluaciones funcionales aplicadas se mostraron susceptibles de perfeccionar. Por ejemplo: fuerza de agarre, encajes y automatización, así como protocolizar el proceso de rehabilitación funcional.

\section{Conclusión}

Para el paciente estudiado, se considera que el uso de la prótesis $3 \mathrm{D}$ puede tener una influencia positiva en el desempeño funcional y la calidad de vida diaria.

\section{Conflictos de interés}

El autor Jorge Zúñiga U, declara haber desarrollado, financiado y proporcionado la prótesis 3D; además, colaboró en el diseño y marco teórico del trabajo.

\section{Referencias bibliográficas}

1. Bethge M, Von Groote P, Giustini A, Gutenbrunner C, The world report on disability: a challenge for rehabilitation medicine. Am J Phys Med Rehabil 2014; 93: S4-11.

2. Krebs D, Edelstein J, Thornby M. Prosthetic management of children with limb deficiencies. Phys Ther
1991; 71: 920-34.

3. Zúñiga J, Katsavelis, Peck J, Stollberg J, Petrykowski M, Carson A, Fernández C. Low-cost 3d-printed prosthetic hand for children with upper-limb differences. BMC Research Notes 2015; 8: 10.

4. Martinot C, Quintero N, Brennetot N, Chapuy M, Fiat M, Fahmy M, Fitoussi F. Latest trends in upper limb prothesis for children with agenesia. Ann Phys Rehabil Med. 2016; Suppl 59: pe11.

5. Bray J. Prosthetic principles, upper extremity amputations: Fabrication and fitting principles. ProstheticsOrthotics Education Program, Division of Orthopedic Surgery, University of California, Los Angeles; 1970. $353 \mathrm{p}$.

6. Davids J, Wagner L, Meyer L, Blackhurst D. Prosthetic management of children with unilateral congenital below-elbow deficiency. J Bone Joint Surg Am. 2006; 88: 1294-300.

7. Scotland T, Galway H. A long-term review of children with congenital and acquired upper limb deficiency. J Bone Joint Surg Br. 1983; 65: 346-9.

8. James M, Bagley A, Brasington K, Lutz C, McConnell $\mathrm{S}$, Molitor $\mathrm{F}$. Impact of prostheses on function and quality of life for children with unilateral congenital below-the-elbow deficiency. Bone Joint Surg Am. 2006; 88: 2356-65.

9. Medical Research Council of the UK: Aids to the investigation of Peripheral Nerve Injuries. Memorando No.45. London, Pendragon House 1976; 6-7.

10. Naranjo J, Escalona P, Solís F, San Martín P. Pauta funcional de mano Bilan 400 points validada en población de 7 a 17 años de edad portadora de discapacidad neuromúsculo-esquelética. Rehabilitación (Madr) 2014; 48: $151-9$.

11. Chesworth BM, Hamilton CB, Walton DM, Benoit M, Blake TA, Bredy H, et al. Reliability and validity of two versions of the upper extremity functional index. Physiotherapy Canada 2014; 66: 243-53.

12. Day H, Jutai J. Measuring the psychosocial impact of assistive devices: the PIADS. Canadian Journal of Rehabilitation 1996; 9: 159-68.

13. Ten Kate J, Smit G, Breedveld P. 3D-printed upper limb prostheses: a review. Disabil Rehabil: Assist Technol 2017; 12: 300-14. 